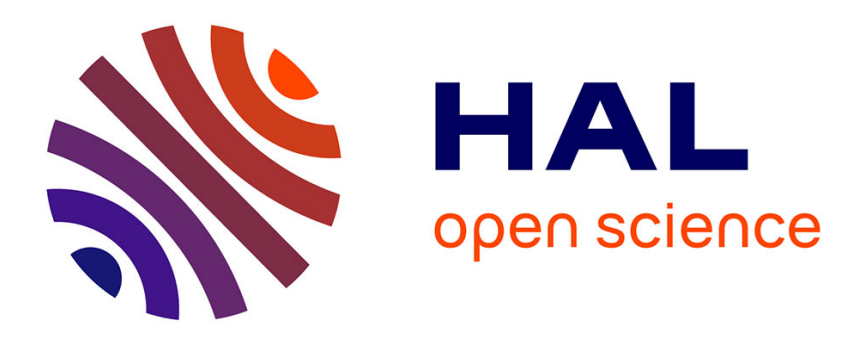

\title{
Adsorption of a polyampholyte chain
}

Jean-François Joanny

\section{To cite this version:}

Jean-François Joanny. Adsorption of a polyampholyte chain. Journal de Physique II, 1994, 4 (8), pp.1281-1288. 10.1051/jp2:1994199 . jpa-00248042

\section{HAL Id: jpa-00248042 https://hal.science/jpa-00248042}

Submitted on 1 Jan 1994

HAL is a multi-disciplinary open access archive for the deposit and dissemination of scientific research documents, whether they are published or not. The documents may come from teaching and research institutions in France or abroad, or from public or private research centers.
L'archive ouverte pluridisciplinaire HAL, est destinée au dépôt et à la diffusion de documents scientifiques de niveau recherche, publiés ou non, émanant des établissements d'enseignement et de recherche français ou étrangers, des laboratoires publics ou privés. 
Classification

Phvstcs Abstracts

$36.20 \mathrm{C}-61.25 \mathrm{H}-68.45$

\title{
Adsorption of a polyampholyte chain
}

\author{
Jean-François Joanny \\ Institut Charles Sadron, 6 rue Boussingault, 67083 Strasbourg Cedex, France \\ (Recenved 24 March 1994, received in final form 5 May 1994, accepted 9 May 1994)
}

\begin{abstract}
We discuss the adsorption of a single polyampholyte chain on a planar solid surface carrying $\sigma$ charges per unit area. We consider the two cases where the interaction between the monomers and the surface is short range (strongly screened) and long range (unscreened). For short range interactions we use the replica trick and a Hartree approximation to show that the chain adsorbs even if th has the same net charge as the surface if the interaction potential is larger than a critical value $V_{\mathrm{c}}=\log f / g$ where $f$ and $g$ are the fraction of monomers having respectively the same charge as the surface and the opposite charge. For long range interactions the adsorption is monitored by the electric field gradient existing in the vicinity of the surface. It occurs if the charge density of the surface is large enough $\sigma a \ell_{\mathrm{B}}>(f-g) /(f+g)^{1 / 2}$ where $a$ is the monomer size and $\ell_{B}$ the so-called Bjerrum length.
\end{abstract}

\section{Introduction.}

A polymer chain comprising charged ionic groups of the same sign (polyelectrolyte) is in general soluble in a solvent with a high dielectric constant such as water because of the strong Coulombic repulsions between the monomers [1]. When two monomers of opposite electrostatic charges are interspersed within the same chain (polyampholyte), the situation is more complex $[2,3]$ : on the one hand, if the total charge does not vanish, the polyelectrolyte effect due to the average Coulombic repulsion between monomers tends to swell the chain and to make it soluble; on the other hand the polyampholyte effect due to the attraction between monomers of opposite charges tends to collapse the chain and to make it insoluble.

The physical properties of neutral polyampholyte chains (with no net electrostatic charge) are dominated by the attractive interactions. It has been observed experimentally $[4,5]$ that if the positively charged and negatively charged monomers are randomly distributed along the polymer backbone neutral polyampholytes are insoluble and can be dissolved only by addition of salt. Non-neutral polyampholytes are soluble if their total charge is large enough.

From the theoretical point of view, the attractions that provoke the phase separation of a polyampholyte solution or the collapse of an individual polyampholyte chain are induced by fluctuations in the charge density similar to those existing in a simple electrolyte solution. In reference [3] these fluctuations were treated at the level of the Debye-Hückel approximation. 
This gives a good qualitative explanation of the experimental observations if the sequence of charges is random.

Another parameter that influences strongly the properties of polyampholytes is the distribution of the charged monomers [6]. A polymer where the positively charged and negatively charged monomers are alternating can be soluble in water whereas a polymer made of the same monomers but randomly distributed along the chain is insoluble. It can be argued that the random polyampholyte is well described by the Debye-Hückel theory which predicts strong attractions and thus a precipitation but that the alternating polyampholyte is equivalent to a polymer carrying dipoles. This leads to a much weaker attraction which can be described by a negative virial coefficient not always large enough to provoke precipitation.

The properties of polyampholyte chains in solution have also been studied by numerical simulation. Alternating polyampholytes behave as neutral polymers and the polyampholyte interactions can be characterized by a negative virial coefficient between monomers [7]. Random polyampholytes have also been simulated [8]; if each chain is exactly neutral, a collapse is observed that is well described by the Debye-Hückel theory. If the chains are only statistically neutral i.e. if they are neutral on average, each chain carries a charge of a given sign, this is found sufficient to overcome the polyampholyte attraction and to swell the polymers. It is however not clear whether this result is valid in the asymptotic limit of infinite molecular weight or if it is a finite size effect.

Recently, experiments have been performed in Strasbourg [9, 10] to study the adsorption of polyampholytes on charged latex particles. The spectacular result that has been obtained is that the polyampholyte solution can adsorb even if its overall charge has the same sign as that of the latex particle.

The purpose of this paper is to study the adsorption of a single random polyampholyte chain on a charged planar surface mimicking the latex particle. In the following section, we discuss the simpler case where the polyampholyte is modelled by a Gaussian polymer chain with two types of monomers some of these being attracted by the surface some being repelled by the surface with the same short range potential. The more realistic case of long range electrostatic interactions is discussed in section 3 at the level of scaling arguments. The final section gives some concluding remarks.

\section{Adsorption of a polyampholyte chain with short range interactions.}

We first study the adsorption of a polyampholyte chain within the framework of an extremely simplified model where the monomers building up the chain do not interact with each other but interact with the adsorbing surface with a short range potential. This would roughly correspond to the case where a large amount of salt has been added to the solution and where the electrostatic interactions are strongly screened. Note however that experiments cannot be performed easily in this limit since the latex particles have a tendency to flocculate. We thus consider here polyampholyte adsorption as equivalent to the adsorption of a neutral random copolymer [11].

The adsorbing polyampholyte comprises $N$ monomers of three types: a fraction $1-(f+g)$ of the monomers are neutral and do not interact with the surface ; a fraction $f$ of the monomers have the same «charge» as the surface and feel a short range repulsive interaction with the surface $k T V(>0)$ and a fraction $g$ of the monomers are attracted towards the surface and feel an attractive potential $-k T V$. (In the case of a polyampholyte in a salt solution with a screening length $\kappa^{-1}$ adsorbing on a plane of charge density $\sigma$, the potential $V$ is equal to $\sigma \ell_{\mathrm{B}} / \kappa$ where $\ell_{\mathrm{B}}$ is the Bjerrum length defined below). The number of charges and their positions along the chain are quenched and imposed by the chemistry. We introduce the charge $q_{1}$ of monomer $i$ equal to one if the monomer is repelled by the surface, -1 if it is 
attracted and 0 if it is neutral. The average value of the charge is $\left\langle q_{1}\right\rangle=f-g$ and if the charges are randomly distributed, the two points correlation function is $\left\langle q, q_{\jmath}\right\rangle=(f+g) \delta_{\imath \jmath}+(f-g)^{2}\left(1-\delta_{\imath \jmath}\right)$ where $\delta_{\imath \jmath}$ is a Kronecker symbol.

For the sake of simplicity we use a lattice model with a lattice spacing $a$. The monomers interact with the surface if they lie in the first layer parallel to the surface. The energy of a given configuration of the chain characterized by the coordinates $z_{l}$ of the monomers perpendicular to the surface is

$$
H=H_{0}+k T V \sum q_{1} \delta_{0}
$$

where $H_{0}=3 k T / 2 a^{2} \sum\left(z_{t+1}-z_{1}\right)^{2}$ is the elastic energy of the Gaussian non-interacting chain.

The partition function is $Z=\sum_{\text {cont }} \exp -\{H / k T\}$ where the sum is extended to all the configurations of the chain. The partition function can be calculated using the replica trick [12] following closely the lines of reference [13]. We introduce $n$ replicas of the chain $z_{1}^{\alpha}$ and calculate $Z^{n}$. This quantity can be averaged over the various realizations of the distribution of charges as

$$
\left\langle Z^{n}\right\rangle=\sum_{\text {cont }} \exp -\left\{\sum_{\alpha} H_{0}^{\alpha} / k T\right\} \prod_{!}\left\{1-(f+g)+f \exp -\left[\sum_{\alpha} V \delta_{0 z_{i}^{\alpha}}\right]+g \exp \left[\sum_{\alpha} V \delta_{0 z_{i}^{\alpha}}\right]\right\}
$$

As a first approximation, we assume that the interaction potential is smaller than $k T(V \ll 1)$ and expand the replica partition function in powers of $V$. This leads to

$$
\left\langle Z^{n}\right\rangle=\sum_{\text {Lon } 1} \exp -A
$$

where the effective free energy is

$$
\begin{aligned}
A=\sum\left\{H_{0}^{\alpha} / k T\right\}+\sum \delta_{0=, a}\left[V(f-g)-V^{2} / 2\left\{(f+g)-(f-g)^{2}\right\}\right]- \\
-V^{2} / 2 \sum_{a \neq \beta,} \delta_{0 z_{1}^{\alpha}} \delta_{0=z_{1}^{\beta}}\left\{(f+g)-(f-g)^{2}\right\} .
\end{aligned}
$$

As in reference [13], we discuss the adsorption of the polymer chain by looking for the ground state of the quantum Hamiltonian

$$
\begin{aligned}
H_{u}=-a^{2} / 6 \sum \frac{\partial^{2}}{\partial z_{\alpha}^{2}}+a \sum_{\alpha} \delta\left(z_{\alpha}\right)\left[V(f-g)-V^{2} / 2\left\{(f+g)-(f-g)^{2}\right\}\right]- \\
\quad-V^{2} / 2 \sum_{\alpha \neq \beta} a^{2} \delta\left(z_{\alpha}\right) \delta\left(z_{\beta}\right)\left\{(f+g)-(f-g)^{2}\right\} .
\end{aligned}
$$

where for convenience we have gone to the continuum limit for the variable $z$ and replaced the Kronecker symbols by Dirac $\delta$ functions.

In the approximation of ground state dominance [14], the partition function $\left\langle Z^{n}\right\rangle$ is equal to $\exp \left(-N n \varepsilon_{0}\right)$ where $n \varepsilon_{0}$ is the energy of the ground state of the Hamiltonian $H_{n}$.

In order to find the ground state. we use a Hartree variational approximation and look for a wave function $\Psi=\prod \phi\left(z_{\alpha}\right)$. In the limit where the number $n$ of replicas goes to zero, the 
Hartree equation reads

$$
\varepsilon_{0} \phi=-a^{2} / 6 \frac{\partial^{2} \phi}{\partial z^{2}}+U(z) \phi
$$

The effective potential in this equation is localized on the surface and proportional to a $\delta$ function, it is given by

$$
U(z)=a \delta(z)\left[V(f-g)-V^{2} / 2\left\{(f+g)-(f-g)^{2}\right\}+V^{2} / 2 a\langle\delta(z)\rangle\left\{(f+g)-(f-g)^{2}\right\}\right]
$$

where the average $\langle\delta(z)\rangle$ is calculated with the ground state wave function

$$
\langle\delta(z)\rangle=\int_{0}^{\infty} \mathrm{d} z \phi^{2}(z) \delta(z)=\phi(0)^{2}
$$

The effective interaction potential between the monomers and the wall is here of purely electrostatic origin. We have thus assumed implicitly that when the charges are not present on the solid surface, it is neutral with respect to the adsorption of the polymer chain it neither repells nor adsorbs it. The adsorbed states of the chain correspond to localized states decaying from the adsorbing surface $\phi(z)=(2 k)^{1 / 2} \mathrm{e}^{-k z}$ where $D=k^{-1}$ is the thickness of the adsorbed chain on the surface. Such solutions exist only if the Hamiltonian has a bound state i.e. if the effective potential $U$ in equation (5) is attractive. This is the case when the potential $V$ seen by the monomers is large enough

$$
V>V_{\mathrm{c}}=\frac{2(f-g)}{(f+g)} .
$$

For a neutral chain $f=g$ and there is no adsorption threshold, the chain always adsorbs even though the average potential acting on the monomers vanishes. The interpretation of this result is clear, the monomers with a charge opposite to that of the surface are in contact with the surface while the monomers with the same charge as the surface are in the loops dangling in the solution and do not feel the repulsive potential which is assumed here to have an infinitely short range. It is important to note that even if the chain is on average repelled by the surface $(f>g)$, it can adsorb if the interaction potential $V$ is large enough.

Within the Hartree approximation, it is possible to find the threshold for adsorption $V_{\mathrm{c}}$ even when the potential is not small as assumed up to now. At the adsorption threshold, the chain is only weakly confined in the vicinity of the interface and the thickness $D$ diverges. Using equation (7), we find that $\langle\delta(z)\rangle=0$ which means that the monomer concentration on the surface is very small. At the level of the Hartree approximation, the coupling between replicas due to the last term in equation (3) vanishes and only averages within one replica are contributing in the vicinity of the adsorption threshold. One can then expand the partition function $\left\langle Z^{n}\right\rangle$ in powers of $V$ neglecting the crossed terms involving two replicas. This gives $\left\langle Z^{n}\right\rangle=\exp -A$ where

$$
A=\sum_{\alpha}\left[\left\{H_{0}^{\alpha} / k T\right\}+U\left(z_{\alpha}\right)\right] .
$$

In the continuum limit. the effective potential $U(z)$ acting on a monomer reads

$$
U(z)=-\log \left\{f \mathrm{e}^{-1}+g \mathrm{e}^{v}+1-(f+g)\right\} a \delta(z) .
$$


The polyampholyte chain adsorbs if this potential is attractive $(<0)$; this leads to an adsorption threshold

$$
V_{\mathrm{c}}=\log \frac{f}{g}
$$

If the overall charge of the adsorbing chain is opposite to that of the surface, there is no adsorption threshold $\left(V_{\mathrm{c}}<0\right)$. When the total charge is small, $f / g \approx 1$ and $V_{\mathrm{c}}$ is small; one can check by expansion around $f=1 / 2$ that this result is in agreement with equations (8). The value of the adsorption threshold $V_{\mathrm{c}}$ has a simple interpretation : $k T \log \frac{f}{g}$ is the contribution to the chemical potential of a monomer of the free energy of random mixing of positively and negatively charged monomers. As explained above, adsorption is possible by putting the attracted monomers in contact with the surface and the repelled monomers away from the surface. If the charge of the monomers were not frozen (as supposed up to now), but annealed this could be done by reducing the entropy of mixing and compensating this penalty by the energy gained upon adsorption of each monomer. For annealed chains we get a threshold given by (11). Within the Hartree approximation we thus obtain the result that the adsorption threshold for the quenched and annealed problems are equivalent. This result has already been conjectured for similar problems [15].

The thickness of the adsorbed polymer chain can be calculated when the potential is small. In this case $f-g \ll f+g$ and we find

$$
k a=3 V(f+g)\left(V-V_{c}\right) .
$$

As the adsorption strength increases, the thickness decreases as $V^{-2}$

For larger values of $V$ equation (5) leads to a saturation of the thickness but the limiting value is reached only for values of $V$ larger than one for which the approximations made break down.

\section{Adsorption of a polyampholyte chain on a charged surface in the absence of salt.}

3.1 ADSORPTION OF A WEAKLY CHARGED POLYELECTROLYTE CHAIN ON A CHARGED SURFACE. - Before discussing the adsorption of a polyampholyte chain, we briefly consider as a pedagogical example the adsorption of a single weakly charged polyelectrolyte chain on a surface of opposite charge [16].

The polymer has $N$ monomers and a fraction $f$ of these are charged. In the bulk, the conformation of this chain can be described in terms of electrostatic blobs [17]. At small length scales, the electrostatic interaction is weak and the statistics of the chain is Gaussian. At larger length scales, electrostatic interactions are dominant and the chain is stretched. The crossover between the two regimes occurs at the length scale $\xi$ where the electrostatic interaction is of the order of the thermal excitation $k T$.

$$
\xi \approx a\left(\ell_{\mathrm{B}} / a\right)^{-1 / 3} f^{-2 / 3}
$$

where $\ell_{\mathrm{B}}=e^{2} / 4 \pi \varepsilon k T$ is the Bjerrum length, $e$ being the elementary charge and $\varepsilon$ the dielectric constant of the solvent (water).

One can then define blobs of size $\xi$ each containing $p=(\xi / a)^{2}$ monomers. The weakly charged polyelectrolyte chain can be viewed as a linear string of blobs of size $\xi$ with a radius $R \sim N / \mathrm{p} \xi \sim N f^{2 / 3}$

The adsorbing surface is an infinite plane with $\sigma$ charges per unit area. In the absence of added salt the concentration of counterions decays as a power law from the surface and all the 
counterions are localized within the Gouy-Chapman length $\lambda \sim 1 / \sigma \ell_{\mathrm{B}}$ from the surface [18]. The electric field created by this plane is $E \approx \sigma e / \varepsilon$ if the distance to the wall is smaller than $\lambda$ and has a small negligible value (decaying as $1 / z$ ) at larger distances. Note that we ignore the effect of the counterions of the polymer.

We consider here only the strong adsorption limit where the adsorbed polyelectrolyte chain is confined within a thickness $D$ from the surface smaller than the blob size $\xi$. At the length scale $D$ the chain remains Gaussian and it is useful to divide the chain into blobs of size $D$ each containing $g_{D}=(D / a)^{2}$ monomers.

We first consider the case where $\lambda$ is larger than $D$. The confinement energy per blob is $k T$ and the confinement energy per monomer is $k T(D / a)^{-2}$. The electrostatic energy of a monomer at a distance $z$ from the surface is $E z$ and on average the electrostatic energy is $k T f \sigma \ell_{\mathrm{B}} D$. The equilibrium thickness of the adsorbed chain is obtained by minimizing the sum of these two energies

$$
D=\left(a^{2} / f \sigma \ell_{\mathrm{B}}\right)^{1 / 3}
$$

It decays with the charge density of the plane as $\sigma^{-1 / 3}$ i.e. more slowly than the GouyChapman, length $\lambda$. The strong adsorption criterion imposes that $\sigma a^{2}>f$.

At large values of the charge density $\sigma$, the Gouy-Chapman length $\lambda$ becomes smaller than the thickness $D$ and only a finite fraction $\lambda / D$ of the monomers feel the electric field. This occurs is $\sigma a \ell_{\mathrm{B}}>f^{1 / 2}$ The electrostatic energy becomes then $k T f \log D / \lambda$. (The logarithmic variation is due do the decay as $1 / z$ of the electric field.) The minimization of the free energy gives an equilibrium thickness of the adsorbed chain independent of the surface charge density $\sigma$

$$
D \approx a / f^{1 / 2}
$$

3.2 AdSORPTION OF A POLYAMPHOLYTE CHAIN. - We now discuss the adsorption of a polyampholyte chain using the same kind of heuristic arguments as for the polyelectrolyte chain. The disorder due to the randomness of the distribution of the charges along the chain is treated by a so-called Imry-Ma [19] argument.

We restrict ourselves to the case where there is no salt and where the polyampholyte is soluble, this is the case if the total charge is large enough. The criterion obtained in reference [3] is

$$
f-g>(f+g)^{3 / 2} \ell_{\mathrm{B}} / a .
$$

We first consider the case where the thickness of the adsorbed chain on the surface is small compared to the Gouy-Chapman length $\lambda$. In a first approximation, the electric field is the constant electric field created by an infinite plane of constant charge density $E=e \sigma / \varepsilon$. All the monomers would then feel the same electric field and if the polyampholyte chain has the same net charge as the adsorbing plane the total electrostatic force is repulsive. However, because of the existence of the counterions of the adsorbing plane, there is an electric field gradient and the electric field decays to zero away from the surface. If the thickness of the adsorbed layer is small, we approximate the field gradient by a constant $E=e \sigma / \varepsilon(1-z / \lambda)$. The electric field is larger close to the surface and energy can be gained by distributing the charges of the polyampholyte chains in such a way that the repelled charges are away from the surface and the attracted charges are closer to the surface. The adsorption results then from a balance between the total electric field that tends to repell the chain from the surface and the electric field gradient that tends to attract the chains towards the surface. 
The average repulsive electrostatic force per monomer is $(f-g) e \sigma / \varepsilon$. In order to estimate the attractive force due to the electric field gradient, we divide the adsorbed polymer chain of thickness $D$ into two layers of thickness $D / 2$. On average each of these layers has the same charge since the charged monomers are distributed perfectly randomly. In order to adsorb the polyampholyte chain a fluctuation of attracted monomers is needed in the layer close to the plane. If the total charge of the chain in this layer is $\delta N$ the attractive force is $-\delta N e \sigma D /(\varepsilon \lambda)$. The charge fluctuation can be calculated by making a blob model of the adsorbed chain. We decompose the chain into Gaussian blobs of size $D$ each containing $g_{D}=(D / a)^{2}$ monomers. In each of these blobs the fluctuation of monomers which are attracted towards the surface within a layer of size $D / 2$ is $\delta g_{D}=(f+g)^{1 / 2} g_{D}^{1 / 2}$ [20]. The total fluctuation for the chain is $\delta N=N \delta g_{D} / g_{D}=N a(f+g)^{1 / 2} / D$. The attractive force per monomer is then $-a(f+g)^{1 / 2} \sigma /(\varepsilon \lambda)$. The total electrostatic force acting on a monomer is

$$
f_{\mathrm{mon}}=k T \sigma \ell_{\mathrm{B}}\left[(f-g)-a(f+g)^{1 / 2} / \lambda\right] .
$$

Adsorption occurs when the force is attractive i.e. if the charge density of the plane is large enough

$$
\sigma a \ell_{\mathrm{B}}>(f-g) /(f+g)^{1 / 2}
$$

Above the adsorption threshold, the attractive electrostatic force is compensated by the elastic force $k T a^{2} / D^{3}$, the thickness of the adsorbed chain is then

$$
D \approx a(f+g)^{-1 / 6}\left(\sigma a \ell_{\mathrm{B}}\right)^{-2 / 3}
$$

As the charge density of the adsorbing plane increases, this thickness becomes larger than the Gouy-Chapman length when $\sigma a \ell_{\mathrm{B}} \approx(f+g)^{1 / 2}$ For larger values of $\sigma$, only a fraction $\lambda / D$ of the monomers feel the electric field. The charge fluctuation in a layer of charge $\lambda$ close to the surface is then $\delta g_{D}=\left[\lambda g_{D}(f+g) / D\right]^{1 / 2}$ for each blob of size $D$ and the total attractive force per monomer is $-k T a(f+g)^{1 / 2}\left(\sigma \ell_{\mathrm{B}}\right)^{1 / 2} D^{-3 / 2}$ This gives a thickness of the adsorbed chain on the plane

$$
D \approx a(f+g)^{-1 / 3}\left(\sigma a \ell_{\mathrm{B}}\right)^{-1 / 3}
$$

\section{Concluding remarks.}

The important result of this paper is that both in the case of short range interactions (strongly screened electrostatics) and in the case of long range interactions, a charged polyampholyte can adsorb on a surface carrying an electrostatic charge of the same sign. The adsorption mechanism is however different in the two cases : for short range interactions the adsorption is due to the redistribution of the charges of the polymer on the surface and an entropy penalty must be overcome : in the case of long range coulombic interactions, the adsorption is due to the gradient in electric field existing in the vicinity of the adsorbing surface. These results are in qualitative agreement with recent experiments.

It is clear that the arguments that we have given are rather rough especially in the case of long range interactions and that a more detailed theory would be needed. Several extensions of our model must also be performed before any quantitative comparison with experiments is possible :

- we have studied here the adsorption of a single chain and not the adsorption of a polyampholyte solution. It is however reasonnable at least qualitatively to assume that if a single chain adsorbs, this is also the case for a solution; 
- we have considered only direct electrostatic interactions and ignored both the non electrostatic interactions and the image charge effect due to the discontinuity of the dielectric constant at the adsorbing surface [21]. The situation that we have discussed is close to the case where the polyampholyte with the same charge sequence is just at the adsorption threshold on a neutral surface $(\sigma=0)$ and where the charge of the adsorbing plane is then increased to a finite value ;

- we have considered a surface with a uniform charge density. In many cases the charge is localized on certain sites. The discreteness of the charges may be important for the adsorption problem ;

- we have considered only random polyampholytes. The precise nature of the distribution of charges is probably important for the adsorption problem. The mechanisms invoked here suggest that no adsorption or at least a much weaker adsorption would occur for perfectly alternating polyampholytes.

Finally, let us mention that our predictions could perhaps be tested directly by computer simulations.

\section{Acknowledgements.}

I am grateful to F. Candau and E. Pefferkorn (I.C.S. Strasbourg) for useful discussions.

\section{References}

[1] Joanny J. F., Leibler L., J. Phys. France 51 (1990) 545.

[2] Edwards S., King P., Pincus P., Ferroelectrics 30 (1980).

[3] Higgs P., Joanny J. F., J. Chem Phys. 94 (1991) 1543.

[4] Corpart J. M., Selb J., Candau F., Polymer 34 (1993) 3873.

[5] Skouri M., Munch J. P., Candau J., Neyret S., Candau F., Marcomolecules 27 (1994) 69.

[6] Wittmer J., Johner A., Joanny J. F.. Europhys. Lett. 24 (1993) 263.

[7] Victor J. M., Imbert J. B., Europhys. Lett. 24 (1993) 189.

[8] Kantor Y., Li H., Kardar M., Phys. Rev. Lett. 69 (1992) 61.

[9] Ouali L., Neyret S., Candau F., Pefferkorn E., in preparation.

[10] The adsorption of polyampholytes has also been studied by Blaackmeer J., Thesis University of Wageningen (1990).

[11] The adsorption of random copolymers has been studied in various other limits see for example Donley J. P., Fredrickson F., Macromolecules 27 (1994) 458 :

Marques C. M., Joanny J. F., Macromolecules 23 (1990) 268.

[12] See for example, Lubensky T., Ill Condensed Matter, R. Balian, R. Maynard, G. Toulouse Eds. (North Holland Amsterdam 1979).

[13] Garel T., Huse D. A., Leibler S., Orland H., Europhys. Lett 8 (1989) 9.

[14] de Gennes P. G., Scaling concepts in Polymer Physics (Cornell University Press Ithaca, 1985).

[15] Nieuwenheuizen T., private communication.

[16] Borisov O., Zhulina E., Birshtein T., J. Phys. II France 4 (1994) 913.

[17] de Gennes P. G., Pincus P., Velasco R. M., Brochard F., J. Phys. France 37 (1976) 1461.

[18] Pincus P., Joanny J. F.. Andelman D., Europhys. Lett. 11 (1990) 763.

[19] Imry Y.. Ma S., Phys. Rev'. Lett. 35 (1975) 1399.

[20] We assume here that $f$ and $g$ are small and that $f-g \leqslant f+g$.

[21] Wittmer J., Joanny J. F., Macromolecules 26 (1993) 2691. 